\title{
Updating DEMs using RADARSAT-1 data
}

\author{
Michael Seymour and lan Cumming
}

\begin{abstract}
We present and analyze an algorithm for the production of accurate digital elevation models (DEMs) using interferometric synthetic aperture radar (InSAR). The algorithm requires minimal manual intervention, as a result of using coarse or low-quality DEMs in the InSAR processing stream. The low-quality DEM data are used to estimate the relative geometry (baseline parameters) of the SAR systems. The baseline parameters are estimated during two different stages of InSAR processing: $(i)$ during interferogram conditioning, where the raw interferogram is preconditioned in preparation for phase unwrapping; and (ii) during production of the final InSAR-updated DEM. Analysis of the algorithm shows that the estimated baseline parameters result in an output InSAR DEM with approximately the same mean and trends in range and azimuth as the input DEM. This is achieved because the new algorithm allows the baseline parameters to absorb errors due to offsets and trends in the auxiliary parameters, such as range distance and satellite altitudes, and in the unwrapped phase. We have demonstrated the ability of the algorithm to improve DEMs of various qualities using RADARSAT-1 InSAR data. The generated DEMs have standard deviations of 12-20 m with respect to a control DEM with an accuracy of $3 \mathrm{~m}$ standard deviation. This represents a two to four times improvement in height accuracy compared with the input DEMs.
\end{abstract}

Résumé. Nous présentons et analysons un algorithme pour la production de modèles numériques de terrain (MNT) de précision utilisant des données interférométriques radar à synthèse d'ouverture (InSAR). L'algorithme requiert une intervention manuelle minimale dû au fait qu'on utilise des MNT de qualité grossière ou faible dans la procédure de traitement InSAR. Les données MNT de qualité réduite sont utilisées pour estimer la géométrie relative (paramètres de référence) des systèmes ROS. Les paramètres de référence sont estimés au cours de deux étapes différentes du traitement InSAR : (i) durant le conditionnement de l'interférogramme où l'interférogramme brut est pré-traité en préparation pour le développement de phase, et (ii) durant la production du MNT final mis à jour par InSAR. L'analyse de l'algorithme montre que les résultats des paramètres de référence estimés constituent un MNT InSAR de sortie ayant approximativement la même moyenne et tendances en portée et azimut que le MNT d'entrée. Ceci est possible grâce au nouvel algorithme qui permet aux paramètres de référence d'absorber les erreurs dues aux décalages et tendances dans les paramètres auxiliaires, comme la distance en portée et les altitudes du satellite, et dans la phase non développée. Nous avons démontré le potentiel de l'algorithme dans l'amélioration des MNT de qualités diverses en utilisant des données InSAR de RADARSAT-1. Les MNT générés donnent des erreurs standard de 12-20 m par rapport à un MNT de contrôle, avec une précision de $3 \mathrm{~m}$ au niveau de l'erreur standard. Ceci représente une amélioration de l'ordre de deux à quatre fois supérieure en précision sur la hauteur comparativement aux MNT d'entrée.

[Traduit par la Rédaction]

\section{Introduction}

In two-pass synthetic aperture radar interferometry (InSAR), the phase difference between registered SAR images is processed to produce digital elevation models (DEMs) (Graham, 1974; Zebker and Goldstein, 1986) or surface motion maps (differential interferometry) (Massonnet et al., 1993). For topography estimation using satellite SAR data, the noise and undersampling in the data complicate the processing. The phase unwrapping procedure is particularly affected (see, for example, Davidson and Bamler, 1999; or Ghiglia and Pritt, 1998). Atmospheric and tropospheric changes between the passes (Tarayre and Massonnet, 1994; Goldstein, 1995; Gray et al., 2000; Hanssen et al., 2000) can also create topographic errors, which can be reduced by processing multiple images (Ferretti et al., 1999). It is important to obtain accurate estimates of the relative position of the SAR sensors to convert the unwrapped phase to topography. The relative-position estimates can be made using manually chosen height control points (Zebker et al., 1994; Wegmüller and Werner, 1997; van der Kooij et al., 1996; Massonnet and Vadon, 1993; Massonnet,
1997; Geudtner and Schwabisch, 1996; Schwabisch, 1998) or DEMs (Massonnet, 1997; Seymour, 1999).

Low-quality DEMs can be used in the InSAR processing stream to minimize manual intervention and reduce the impact of low signal to noise ratio (SNR) on InSAR data processing (Seymour, 1999; Eineder, 2003). In our algorithm, the lowquality DEMs are used to precondition the interferogram in preparation for phase unwrapping and to estimate the final geometry parameters required for converting the unwrapped phase to topographic height. We call these two procedures interferogram conditioning and DEM updating.

In the present paper, we embed these procedures in a complete InSAR processing algorithm and apply them to a difficult RADARSAT-1 dataset that has very low coherence. We have also applied the algorithm to European Remote-sensing Satellite (ERS) tandem mission data (Seymour and Cumming,

Received 1 October 2003. Accepted 21 May 2004.

M. Seymour ${ }^{1}$ and I. Cumming. Department of Electrical and Computer Engineering, University of British Columbia, Vancouver, BC V6T 1Z4, Canada.

${ }^{1}$ Corresponding author (e-mail: michael.seymour@eads.com). 
1997), but we report only the RADARSAT-1 results here, as they portray the algorithm under more difficult conditions. The first section of the paper gives an overview of the InSAR processing algorithm, and the second section discusses algorithm performance issues, including achievable DEM accuracies and the effect of using an input DEM as a template for processing. The third section describes the RADARSAT-1 scene and the DEM data used in the experiments. The results of the experiments are given in the fourth section, where we measure how much the coarse DEMs have been improved by the information in the InSAR data.

\section{Overview of DEM refinement algorithm}

To compute terrain height from the interferometric data, the relative positions of the SAR sensors must be known precisely, often within millimetres. We refer to the required geometric parameters as baseline parameters. We have developed two procedures for estimating these baseline parameters using lowquality DEM data (Seymour, 1999), and the present paper deals with an analysis of their accuracy. The first procedure operates on wrapped phase and has the advantage of not being affected by phase unwrapping errors. The second procedure works on unwrapped phase and serves to refine the baseline estimates obtained from the first procedure.

The complete algorithm for InSAR processing using existing low-quality DEMs consists of three main parts, which are outlined in Figure $\mathbf{1}$ and described in the following subsections.

\section{Interferogram conditioning using an existing DEM}

The DEM data are first registered to the InSAR data using the existing satellite position information. The baseline parameters are then estimated by an iterative, nonlinear optimization procedure in which the following steps are embedded: $(i)$ generate a "model interferogram" from the input DEM and the best current estimates of the baseline parameters; (ii) subtract the model interferogram from the actual interferogram, which forms a "residual interferogram" representing topography missing from the input DEM, plus system noise; and (iii) iterate on the baseline parameters until the frequency of the strongest spectrum component in the residual interferogram is as close to zero as possible.

The baseline parameters derived during this modelling procedure are generally not accurate enough for topographic estimation but are adequate for the immediate objective of conditioning the interferogram for phase unwrapping. Removing the model interferogram phase is a type of "flattening" operation that removes much of the complexity from the measured interferogram. The useful information that remains in the residual interferogram has a lower bandwidth and is centred on zero frequency, which allows a more aggressive form of filtering (phase smoothing) to be applied. Lastly, after filtering, the residual interferogram is subsampled to reduce the volume of data that need unwrapping.

\section{Phase unwrapping}

We unwrap the phase using Flynn's (1997) weighted minimum discontinuity method. Binary weights are generated from a combination of coherence magnitude thresholds, image magnitude or SNR thresholds, and limits on the local density of residues. The minimum discontinuity algorithm can be

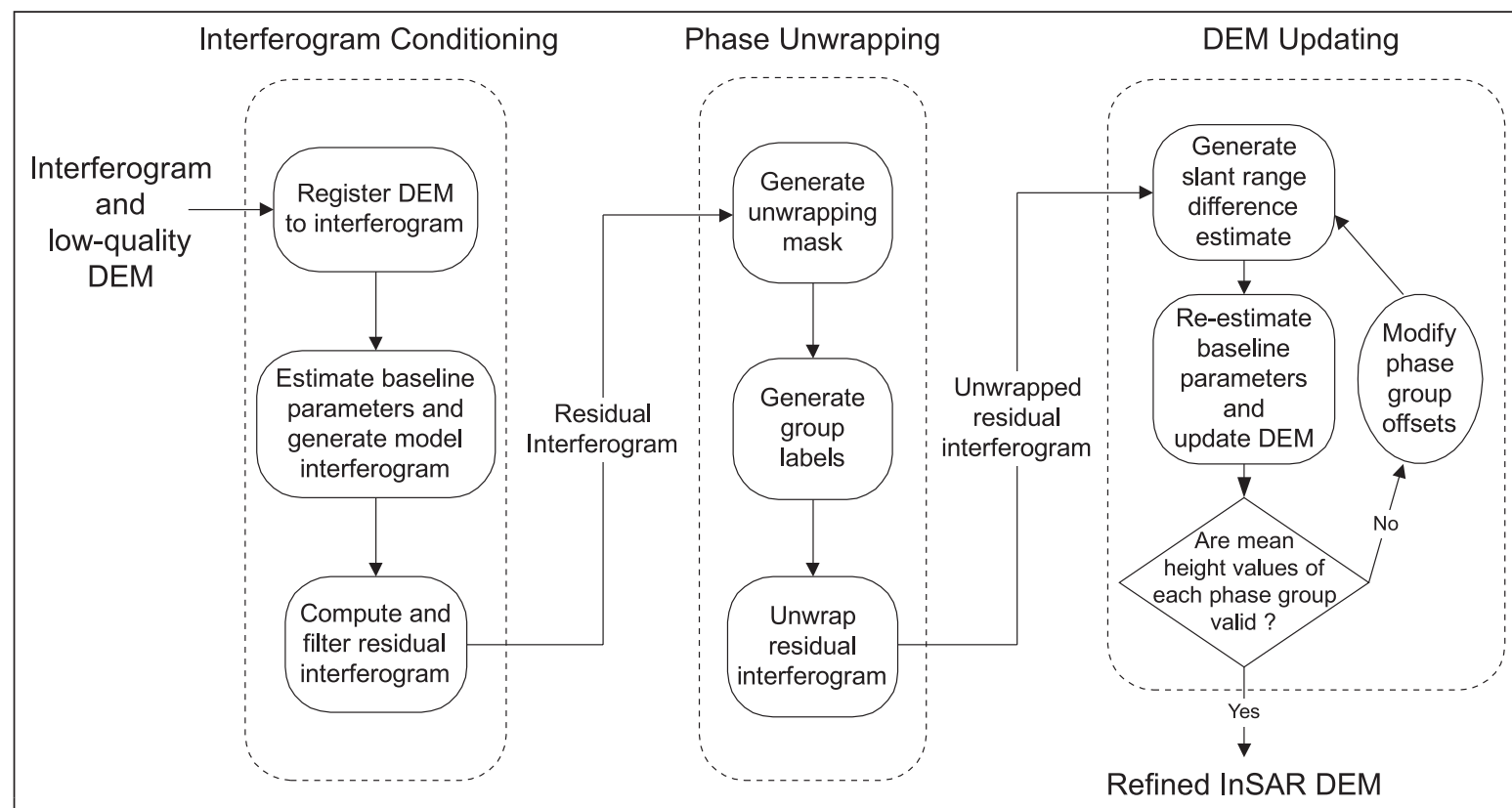

Figure 1. Outline of InSAR processing algorithm using existing DEM data to refine the satellite geometry, improve phase unwrapping, and update the DEM. 
initialized with a partially unwrapped phase estimate to facilitate the processing. We used the unwrapped phase from a weighted least-squares phase unwrapping algorithm (Ghiglia and Romero, 1994) plus the residual wrapped phase as the initial phase estimate.

For two-pass satellite InSAR, there can be instances where the unwrapping weights segment the interferogram into distinct groups of pixels. Based on the weights alone, we assign a "group" label to each pixel so that pixels belonging to the same contiguous phase surface can be recognized and processed together during the DEM updating.

\section{DEM updating}

The initial stage of the DEM updating procedure combines the unwrapped residual phase and the interferogram model to generate an estimate of the phase difference arising from the measured interferogram. The phase difference is converted to an equivalent slant range difference, which is used with the coarse input DEM to reestimate the baseline parameters, using a second iterative nonlinear optimization. These estimates are more accurate than those from the modelling step because the interferogram has been filtered and unwrapped. The input DEM is then refined using the phase information of the residual interferogram and the improved geometry. If there are multiple unwrapped phase groups, the phase offset of each unwrapped phase group can be checked against the input DEM. If there is an inconsistency, the phase offset of the unwrapped group can be adjusted and the process for DEM updating reapplied.

\section{Algorithm performance}

Relatively large normal baselines are needed in satellite InSAR to reduce the sensitivity to phase noise and produce good quality topographic estimates. With large baselines, however, the interferogram conditioning procedure does not estimate baseline parameters with sufficient accuracy to generate accurate InSAR heights, for the following reasons. The iterations of the interferogram conditioning procedure rely on estimating the average frequency of the residual interferogram. When the normal baseline is relatively large, the average frequency component of the residual phase is difficult to recognize and the resulting baseline estimates are not reliable. However, despite this lack of baseline accuracy that sometimes occurs at this stage of the algorithm, the interferogram conditioning procedure still yields a residual interferogram that is much easier to filter and phase unwrap than the usual flatearth corrected interferogram. Therefore, the interferogram modelling algorithm can be considered a data conditioning stage rather than a methodology for producing accurate baseline parameter estimates. In contrast, the DEM updating iterations $d o$ provide baseline estimates that are generally accurate enough to obtain good height estimates.

In the following two subsections, we review the major factors of error in the output height estimates that arise from using coarse DEMs to guide the estimation of the baseline geometry. The two major sources of error are phase noise and geometry errors.

\section{Phase errors}

In two-pass InSAR, errors in the phase come from decorrelation (Zebker and Villasenor, 1992; Prati and Rocca, 1992) and propagation errors (Goldstein, 1995; Tarayre and Massonnet, 1996; Hanssen et al., 2000). Decorrelation errors include effects such as system noise, the effect of local slope, and misregistration. The dispersion of height errors $\left(\sigma_{\mathrm{h}}\right)$ is related to the phase noise $\left(\sigma_{\text {phase }}\right)$ via

$\sigma_{\mathrm{h}}=\frac{\lambda}{4 \pi} \frac{x}{B^{\perp}} \sigma_{\text {phase }}$

where $\lambda$ is the wavelength corresponding to the SAR centre frequency, $x$ is the ground range, and $B^{\perp}$ is the normal baseline.

Generally, decorrelation errors can be filtered relatively easily because they can be treated as uncorrelated noise from pixel to pixel. However, propagation errors are generally more difficult to treat as they are long-wavelength phenomenon. Propagation errors result from the changing speed of radar waves due to inhomogeneities in the atmosphere that vary from pass to pass. For low earth orbiting SAR satellites, the two layers causing difficulties are the ionosphere (Gray et al., 2000) and the troposphere (Hanssen et al., 2000).

A change in the free electron density in the ionosphere changes the speed of the radar waves, resulting in an error in the reconstructed interferogram phase. The ionospheric electron density depends on latitude, solar activity, and geomagnetic activity and varies with a daily cycle. The electron density tends to be a long-wavelength feature, which causes a small ramp in the interferogram phase over $100 \mathrm{~km}$. However, ionospheric activity, particularly near the poles during strong magnetic storms, is thought to be the cause of more localized events (Gray et al., 2000) that manifest themselves as streaks in the azimuth direction of images. The size of these events is in the order of kilometres at C-band, and the streaks can extend up to several hundred kilometres along-track. One potential method of mitigating these features is to use the azimuth registration relations to estimate the amplitude of the ionospheric disturbances (Mattar and Gray, 2002).

In the troposphere, the propagation velocity of the radar energy varies with temperature, pressure, and relative humidity. These parameters vary with the meteorology (Hanssen et al., 2000) and also with the amount of relief in the scene (Dupont, 1997). The effect of local weather conditions, particularly weather fronts, can cause large errors (up to four fringes have been found at C-band (Hanssen, 1997)). In addition, large height variations in the scene can cause significant variation in the amount of atmosphere traversed by the radar energy, leading to atmospheric phase errors that are correlated with topography.

One method of treating the problem of atmospheric phase errors is to process a number of suitable scenes together and use 
the correlated nature of the atmospheric phase noise to filter the phase errors (Ferretti et al., 1999). Alternately, one can try to choose scenes with calm meteorological conditions (i.e., absence of storms or squalls) to minimize the impact of possible atmospheric artifacts. Meteorological conditions at the time of the SAR data collection are usually publicly available from national meteorological organizations. In general though, the effect of atmospheric phase errors can be minimized by treating data coming from an interferometer with the largest possible normal baseline, as the amount of atmospheric phase change is scaled by the inverse of the normal baseline. RADARSAT- 1 fine mode data are particularly suitable for producing InSAR height estimates (Mattar et al., 1999) in the presence of atmospheric phase noise because the maximum perpendicular baseline can be relatively large (about $5 \mathrm{~km}$ ) (Vachon et al., 1995), leading to less sensitivity to phase errors. However, the time between repeat cycles ( 24 days) and the impact of larger baselines leads to noisy data that must be filtered aggressively to obtain good results.

\section{Geometry errors}

Geometric processing errors are related to errors in the baseline orientation and magnitude. Errors in each of these parameters create approximately linear trend errors in the output DEM. These errors are not colinear, so different combinations of baseline parameter errors can actually yield very similar height errors. As the baseline parameter errors may also vary independently in azimuth, the net result is an approximately bilinear error surface. The bilinear nature of the output height errors means that the baseline parameter estimates can converge to erroneous values in such a way that the offset baseline estimates "absorb" errors that have a similar form. For example, errors of up to $50 \mathrm{~m}$ in the satellite altitude, phase trends of the order of one to two cycles across the scene, or several ambiguities in the phase unwrapping offset can be absorbed by modified baseline parameter values, without significantly affecting the accuracy of the output DEM (Seymour, 1999). For this reason, the precise accuracy of the baseline parameters is not necessarily a useful parameter to estimate. Rather, it is more interesting to consider the effects of input DEM errors on the output DEM directly.

The least-squares minimization of output height errors with respect to the input DEM forces the output DEM to take on the apparent offset and trend errors in the input DEM. The apparent height errors are a combination of the effects of the input DEM errors and the height errors due to uncorrelated interferogram phase noise. If we consider the ideal case of no errors other than independent, zero mean phase noise errors, the baseline estimates will converge (in the statistical mean sense) to the true values. Coarse DEM values are not accurate, however, and contribute a source of error that must be taken into account during the minimization process. We analyzed this effect by considering the fit of a bilinear function to noise samples modelling the influence of DEM noise on baseline parameter errors and, finally, on the output height (see Appendix A).
Even if the input DEM has insignificant mean and trend errors, the output DEM will still have some error due to input DEM noise and uncorrelated interferogram phase noise. In the absence of phase errors, the mean and trend errors of the output DEM can be assessed from the statistics of the input DEM alone. If phase errors are present, the mean and trends of their associated height errors will likewise be absorbed by the baseline errors, leaving only the effects of local phase errors in the output DEM. In the case of some of the more extreme values of tropospheric phase errors, this means that the effective offsets and trends of the tropospheric phase errors are absorbed by the baseline parameter errors. However, the residual phase error will affect the output DEM, both in a direct noise component and in the variability of the output fit.

\section{Output DEM height errors}

We analyzed the performance of the algorithm in terms of two criteria, namely standard deviation of the output height errors and standard deviation of the integrated slope of the output height errors (see Appendix A). The first performance parameter is a common measure of DEM performance. However, we found that the standard deviation of the output InSAR height errors was not particularly sensitive to the slope errors, so we measured the slope errors explicitly by calculating the standard deviation of the difference between the height errors at near range and at far range.

The second performance parameter measures tilt errors in the DEM to highlight the particular characteristics of the use of coarse DEMs in the processing. We assumed that the two main sources of error, input DEM errors and interferogram noise, were independent random variables, keeping in mind that the fitting algorithm drives the output DEM to roughly the same apparent trend and mean as the input DEM. The resulting output height variance is

$\sigma_{\mathrm{h}}^{2}=\frac{3}{\mathrm{NrNa}}\left(\sigma_{\mathrm{DEM}}^{2}+\sigma_{\psi}^{2}\right)+\sigma_{\psi}^{2}$

where $\sigma_{\mathrm{h}}$ is the standard deviation of height error in the updated $\mathrm{DEM} ; \sigma_{\mathrm{DEM}}$ is the standard deviation of height error in the input DEM; $\sigma_{\Psi}$ is the updated DEM height standard deviation due to interferogram phase noise; and $\mathrm{Nr}$ and $\mathrm{Na}$ are the number of range and azimuth samples processed, respectively.

The first term on the right-hand side of Equation (2) is the effect of the input DEM and the baseline parameter errors on the output height variance. It is a function of both the level of interferogram phase noise and input DEM errors. The second term is the average height error directly due to noise in the unwrapped phase.

To use Equation (2), the baseline parameters must be known to convert the interferogram phase noise to equivalent height noise. For evaluating the suitability of a DEM for use with the DEM updating algorithm, however, it is useful to consider the effects of the input DEM errors alone by assuming noise-free 
unwrapped phase (i.e., $\sigma_{\Psi}=0$ ). The output DEM variance due to random errors in the input DEM alone is

$$
\sigma_{\text {base }}^{2}=\frac{3}{\mathrm{NrNa}} \sigma_{\mathrm{DEM}}^{2}
$$

This equation provides an estimate of the error of the estimated interferometric height and serves as a preliminary guide to the output DEM accuracy without considering interferogram phase noise.

The contribution of baseline errors to output DEM errors has the form of a bilinear surface, in the absence of interferogram

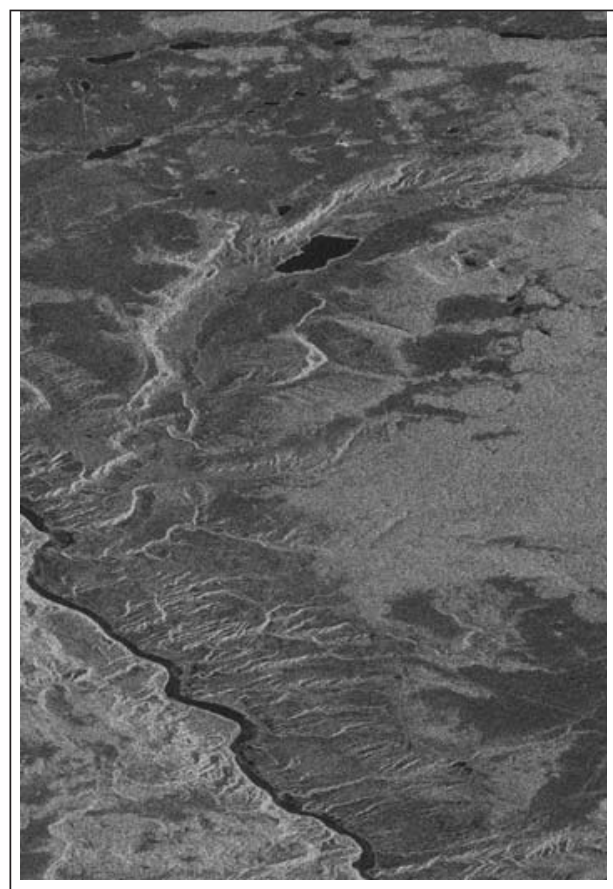

(a) SAR image

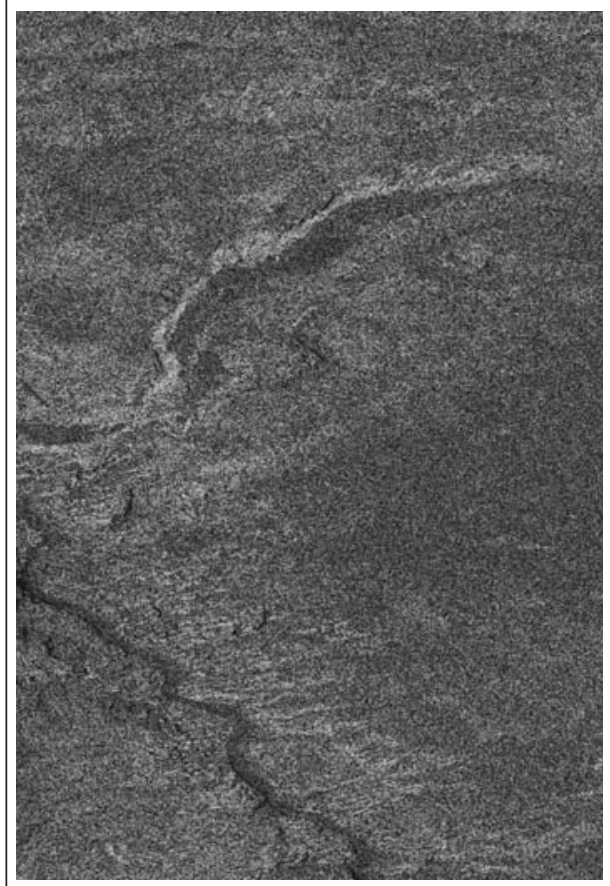

(c) Coherence magnitude

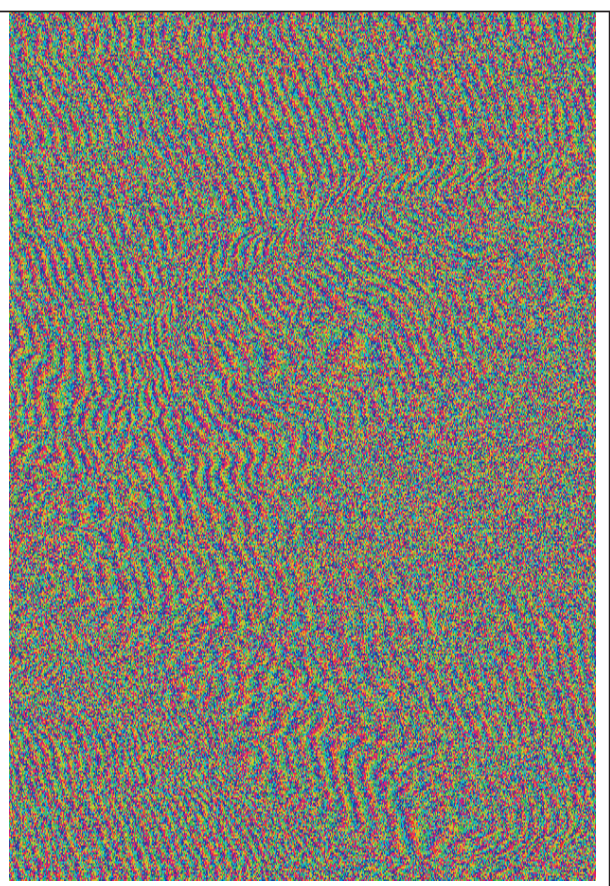

(b) Raw interferogram phase

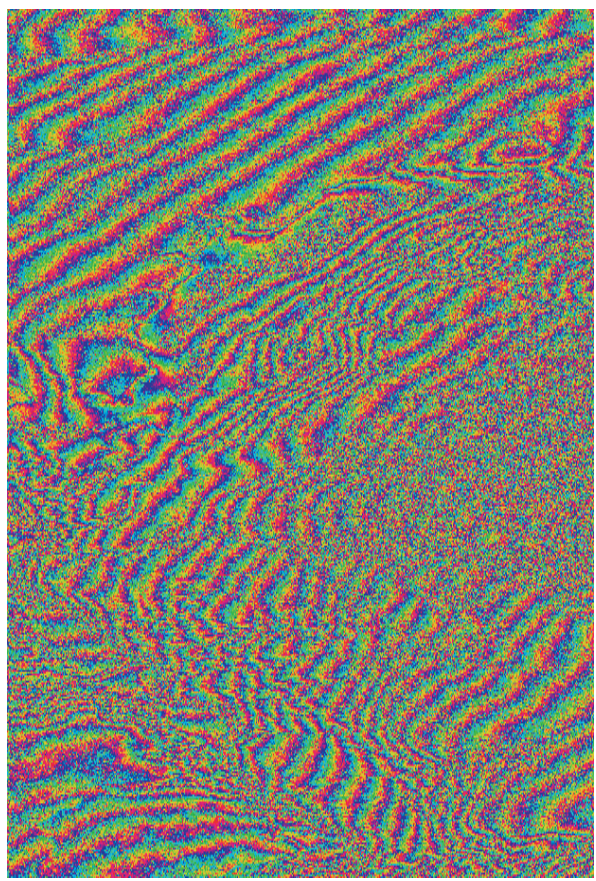

(d) "Flattened" phase

Figure 2. Summary of RADARSAT InSAR data. The flattened interferogram phase was generated by removing the dominant range frequency from the raw interferogram phase (i.e., flat-earth flattening). 
phase noise. As previously mentioned, we characterized this error by evaluating the variance of the height error difference across the swath:

$\sigma_{\text {swath }}^{2} \approx \frac{48}{\mathrm{Nr} \mathrm{Na}} \sigma_{\mathrm{DEM}}^{2}$

This gives an estimate of the size of trend errors across the data swath and is useful for characterizing the relative error performance of the InSAR DEM.

\section{Experimental data}

To illustrate the performance of the algorithm, we performed experiments with a challenging interferogram generated from RADARSAT-1 data. The radar scene is described and the DEM data used in the processing are reviewed in this section. The experimental results of the InSAR processing algorithm are described in a subsequent section.

\section{Radar scene}

The Chilcotin area of British Columbia was chosen as a test site because the topography is very challenging and yet the temporal decorrelation is not so bad as to completely eliminate coherence. The scene has height variations of up to $1400 \mathrm{~m}$, and the Fraser Canyon in the lower left of the image has steep topography that induces some layover and shadowing (see Figure 2a). The river itself is large enough to produce an incoherent strip that subdivides the interferogram into several distinct areas (see Figure 2c). The RADARSAT data cover approximately $10 \mathrm{~km}$ in range and $30 \mathrm{~km}$ in azimuth. This represents about $12 \%$ of the scene from the RADARSAT- 1 fine beam 3, whose parameters are given in Table 1. All images are oriented approximately north up.

Two passes were collected on 24 April (orbit 7675) and 18 May 1997 (orbit 8018) at a local time of 6 a.m. The normal baseline calculated from the RADARSAT orbit data was $200 \mathrm{~m}$, well within the preferred range for RADARSAT fine beam data (the ambiguity height is $80 \mathrm{~m}$ ). The estimated contribution of the baseline parameter errors to the output InSAR DEM error was calculated from Equations (3) and (4) and is shown in Table 2.

The Chilcotin area is usually dry with limited vegetation, so some portions of the scene can provide reasonable repeat-pass interferometric coherence, despite the 24 day observation interval. The RADARSAT interferogram has a rather low average coherence magnitude of 0.35 (see Figure 2c). This fact, combined with the somewhat large baseline of $200 \mathrm{~m}$, adds to the challenge of the dataset.

\section{DEM data}

DEMs of three different qualities were available for the scene, as shown in Figure 3. Each has a different resolution and accuracy, as summarized in Table 3. The gray scale for all the
Table 1. Nominal RADARSAT

fine beam 3 parameters.

\begin{tabular}{ll}
\hline Incidence angle $\left(^{\circ}\right)$ & 41.6 \\
$\quad$ Near range & 44.2 \\
$\quad$ Far range & \\
Slant range $(\mathrm{km})$ & 1020 \\
$\quad$ Near range & 1060 \\
$\quad$ Far range & \\
Swath width $(\mathrm{km})$ & 50 \\
$\quad$ Ground range & 40 \\
$\quad$ Slant range & 0.056 \\
Wavelength $(\mathrm{m})$ & 807 \\
Altitude $(\mathrm{km})$ & \\
\hline
\end{tabular}

DEM images goes from 0 to $1100 \mathrm{~m}$. The DEMs include the following:

(1) TRIM DEM - The TRIM digital dataset is a 1997 product of the Province of British Columbia that consists of a DEM and other cartographic information, such as planimetric features and land cover (Surveys and Resources Mapping Branch, 1992). The DEM was generated using photogrammetric techniques. Elevation data were captured manually in a loose grid pattern with nominal spacing of $100 \mathrm{~m}$ in flat areas and $75 \mathrm{~m}$ in areas of higher relief. The height accuracy of the sampled data points is $5 \mathrm{~m}$ at $90 \%$ confidence (LE90), with a planimetric error of $12 \mathrm{~m}$ circular error at $90 \%$ confidence (CE90).

(2) DTED-1 DEM - The DTED-1 DEM is a discontinued product from the Government of Canada, generated by digitizing hard-copy maps produced using 1950s era mapping technology. It is a gridded dataset with sample spacing of 3 arc seconds, or approximately $90 \mathrm{~m}$. The original hard-copy map is a 1 : 50000 scale map named Noaxe Creek, number 9202. The nominal positional accuracy of the digitized data is $150 \mathrm{~m}$ standard deviation; the nominal vertical accuracy of the data is $100 \mathrm{~m}$ standard deviation (J. Pinard, personal communication, 2000).

(3) GTOPO30 DEM - The GTOPO30 DEM is a project of the United States Geological Survey (USGS) that provides publicly available, coarse, low-resolution datasets suitable

Table 2. Summary of the contributions of the estimated baseline parameter errors to output InSAR DEM accuracy for RADARSAT data.

\begin{tabular}{lllll}
\hline & $\begin{array}{l}\text { No. of } \\
\text { azimuth } \\
\text { samples }\end{array}$ & $\begin{array}{l}\text { No. of } \\
\text { range } \\
\text { samples }\end{array}$ & $\begin{array}{l}\sigma_{\text {base }} \\
(\mathrm{m})\end{array}$ & $\begin{array}{l}\sigma_{\text {swath }} \\
(\mathrm{m})\end{array}$ \\
\hline TRIM & 300 & 100 & 0.03 & 0.12 \\
DTED & 333 & 111 & 0.30 & 0.65 \\
GTOPO30 & 30 & 10 & 8.60 & 30.90 \\
\hline
\end{tabular}

Note: The error estimates were calculated using the DEM error statistics of Table 3 . 
Table 3. Summary of DEM characteristics for the Chilcotin test.

\begin{tabular}{lccl}
\hline DEM & $\begin{array}{l}\text { Sample } \\
\text { spacing }(\mathrm{m})\end{array}$ & $\begin{array}{l}\text { Vertical } \\
\text { accuracy }(\mathrm{m})\end{array}$ & $\begin{array}{l}\text { Horizontal } \\
\text { accuracy }(\mathrm{m})\end{array}$ \\
\hline TRIM & $75-100$ & 5 (LE90) & 12 (CE90) \\
DTED-1 & 90 & $30($ LE90) & 50 (CE90) \\
GTOPO30 & 1000 & $86(\mathrm{rms})$ & na \\
\hline
\end{tabular}

Note: CE90 and LE90, 90\% confidence; na, not available. for modelling large-scale processes (http://edcdaac.usgs. gov/gtopo30/gtopo30.html). A similar dataset is available from the Committee on Earth Observation Satellites (CEOS) Global Land One-Km Base Elevation (GLOBE) project (http://www.ngdc.noaa.gov/seg/topo/globe.shtml). The GTOPO30 DEM was generated by combining various sources of DEM data to generate a composite DEM with data postings of $30 \mathrm{arc}$ seconds (approximately $1 \mathrm{~km}$ ). The

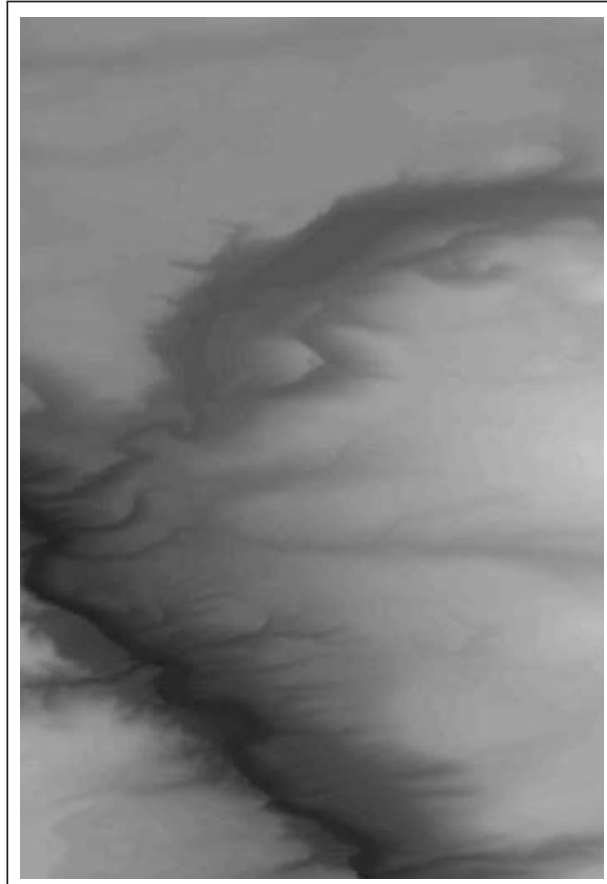

(a) TRIM

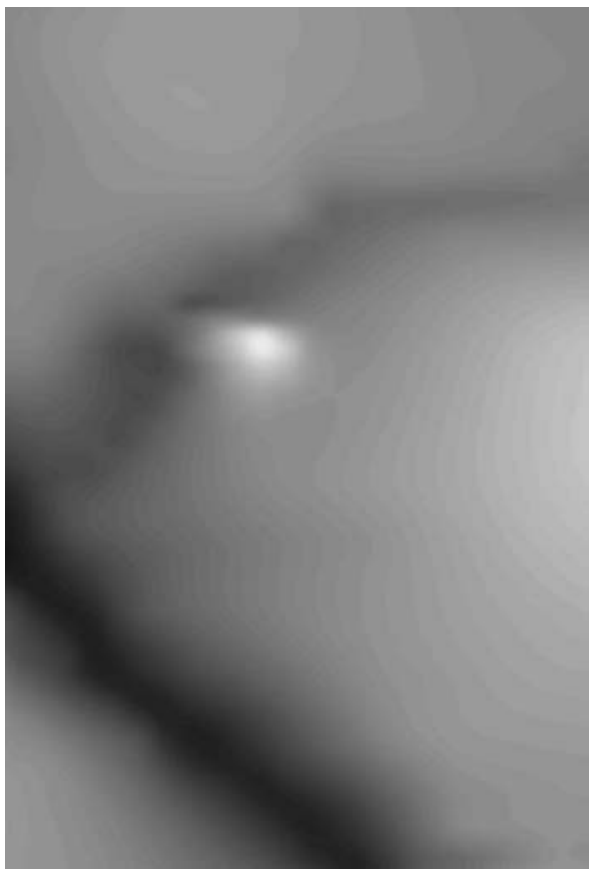

(c) GTOPO30

Figure 3. Input DEMS used for RADARSAT experiments. The DEMs are resampled to SAR slant range coordinates.

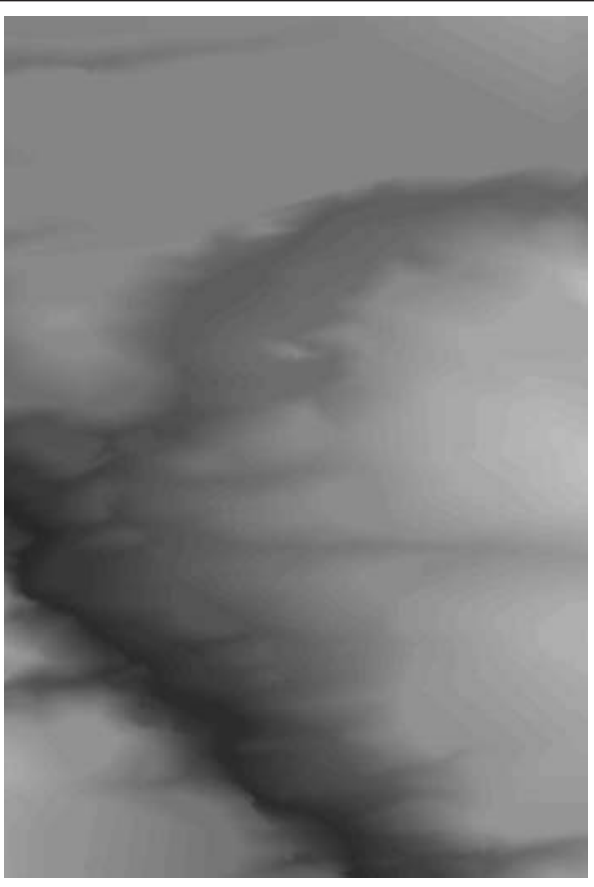

(b) DTED-1 
Vol. 30, No. 6, December/décembre 2004
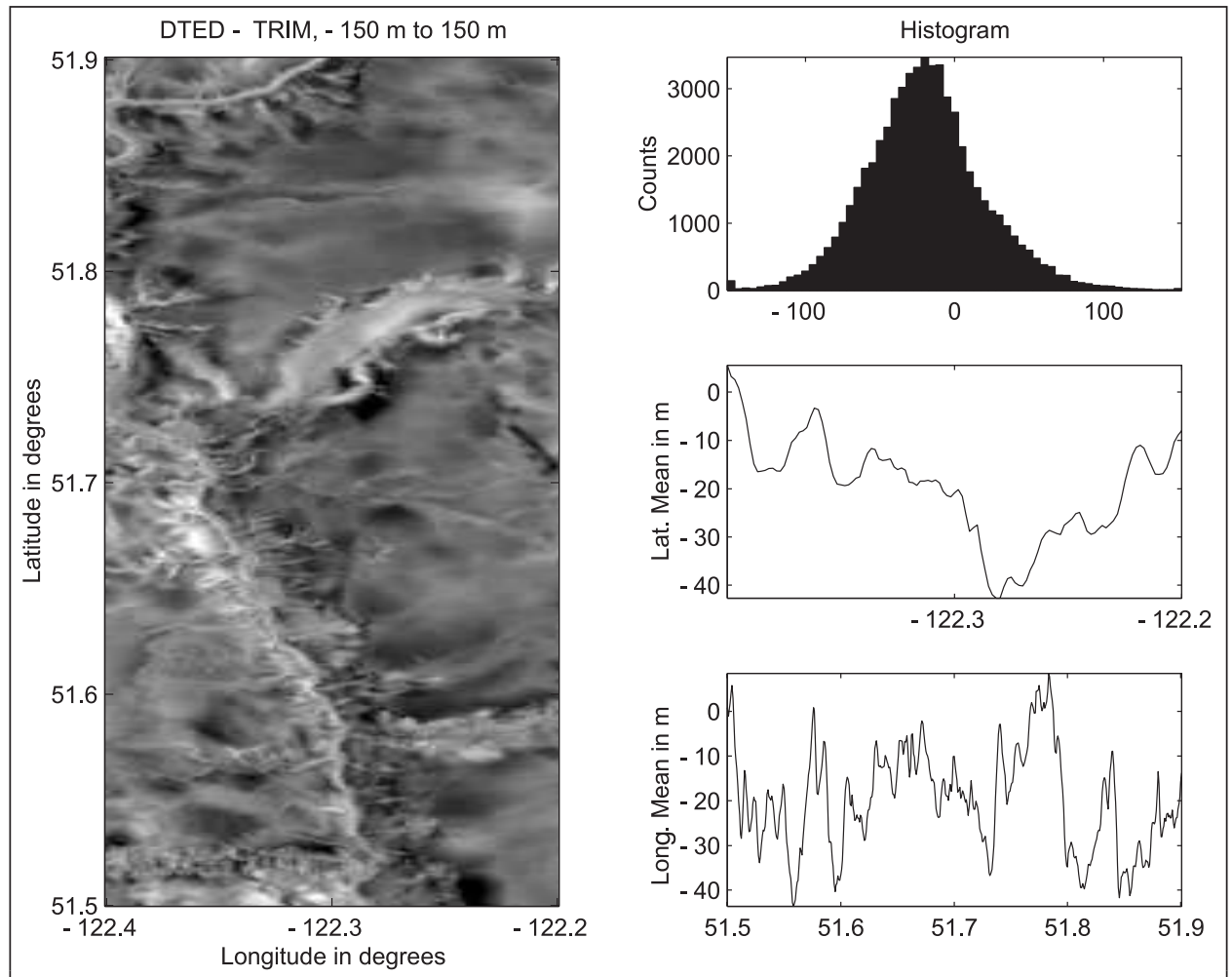

Figure 4. Summary of height differences between the DTED-1 and TRIM data.
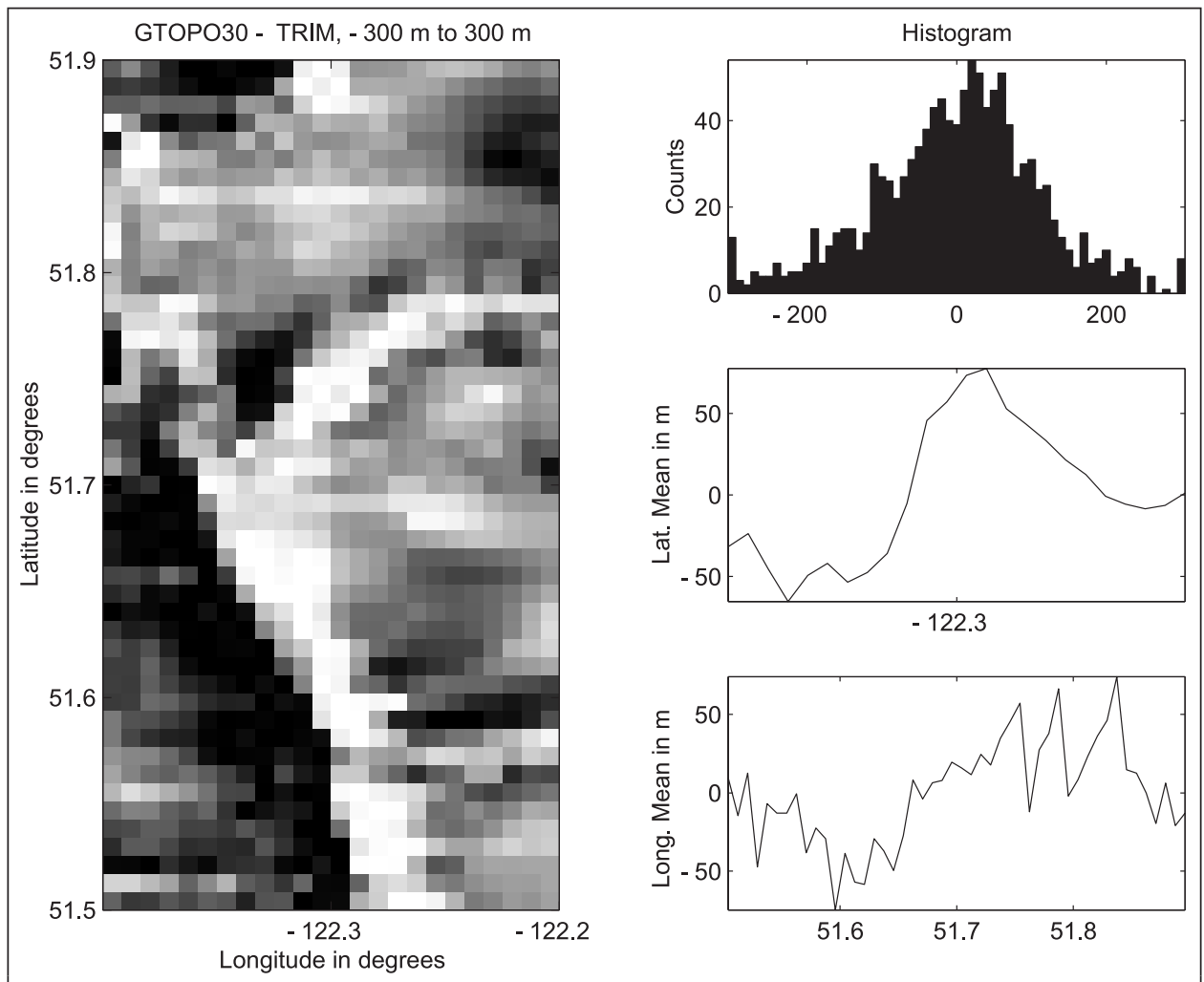

Figure 5. Summary of height differences between the GTOPO30 and TRIM data. 


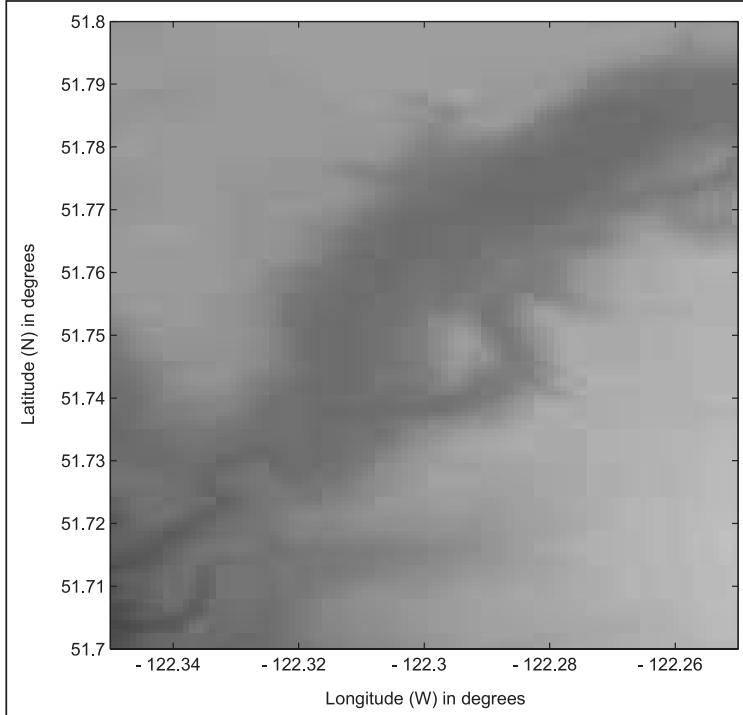

(a) TRIM

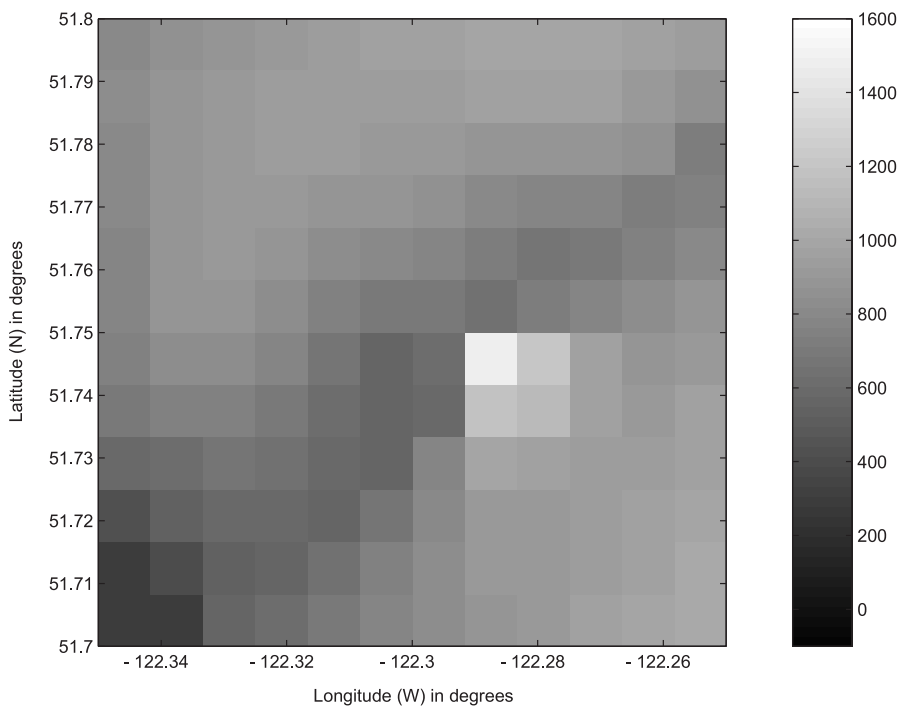

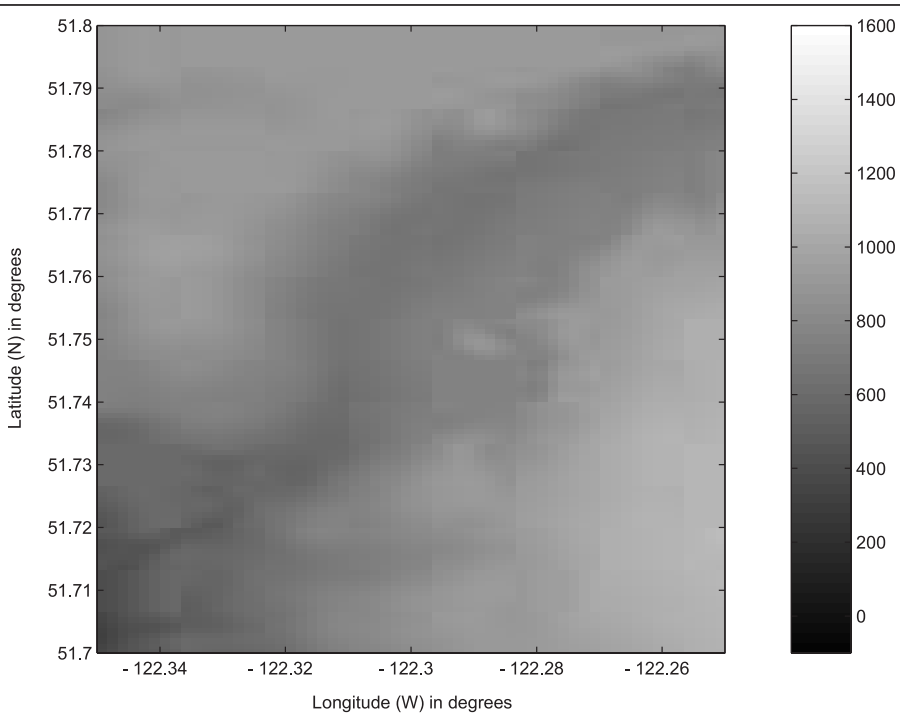

(b) DTED-1

(c) GTOPO30

Figure 6. Zoomed DEMs of the Chilcotin, British Columbia, test site. The artifact in the GTOPO30 DEM can be clearly seen.

accuracy of the GTOPO30 has an estimated root mean square (rms) error of approximately $86 \mathrm{~m}$ for the Chilcotin area, which agrees with other assessments (Gesch, 1998).

The large topographic variation of the terrain can be seen in Figure 3, especially in the river canyons. The difference in resolution and detail between the DEMs can be seen by comparing the different panels in Figure 3.

Because of the higher accuracy of the TRIM DEMs, we use this dataset as an estimate of ground truth in the following analysis. The difference between the DTED-1 and TRIM data interpolated to the same sample positions has a standard deviation of $40 \mathrm{~m}$ with a mean offset of $-20 \mathrm{~m}$, as shown in Figure 4. Note also that there is a trend in the errors from west to east, cutting across the Fraser Canyon. As mentioned in the overview section, both the mean offset and the trend errors will be reflected in the output InSAR heights based on the DTED-1 data. The mean offset and trend errors are probably artifacts of the map from which the digital data were scanned, or mistakes in the interpretation of the parameters of the digital data, such as a wrong datum.

For the GTOPO30 data in Figure 5, the standard deviation of the difference between the GTOPO30 data and the TRIM data interpolated to the GTOPO30 grid is $117 \mathrm{~m}$, with a mean error of $-0.1 \mathrm{~m}$. There is evidence of a trend in errors from east to west, similar to those of the DTED-1 DEM data but reversed in polarity.

Closer examination of a small area of each DEM gives more information on the relative magnitude and characteristics of the DEM height errors. Looking at the region centred at $51.75^{\circ} \mathrm{N}$ and $-122.3^{\circ} \mathrm{W}$, for example, we can see significant differences 
between the DEMs in Figure 6. In the higher resolution TRIM data, it is evident that there is a small "hill" centred at this location. In the DTED data, the hill is not so prominent. Errors of this type can occur at the edges of areas processed at different times during the digital map production. In the GTOPO30 data, there is a large "spike" of $650 \mathrm{~m}$ in the DEM values. We left this anomalous data point in the GTOPO30 dataset to test the robustness of the DEM updating algorithm.

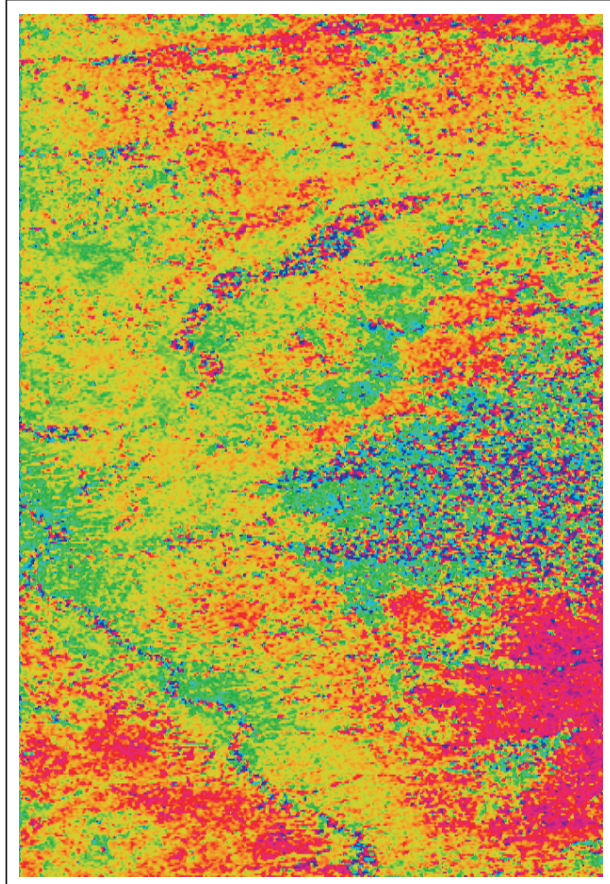

(a) Using TRIM DEM

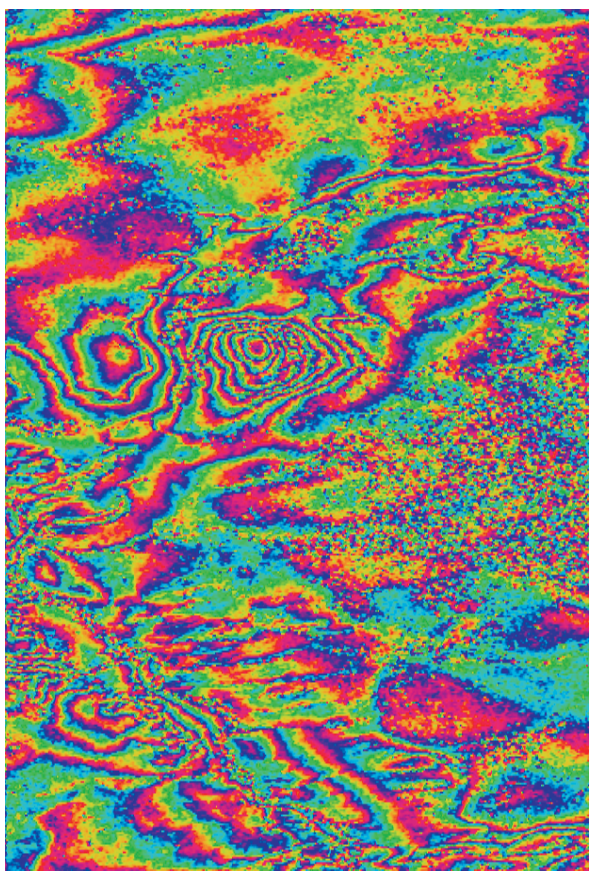

(c) Using GTOPO30

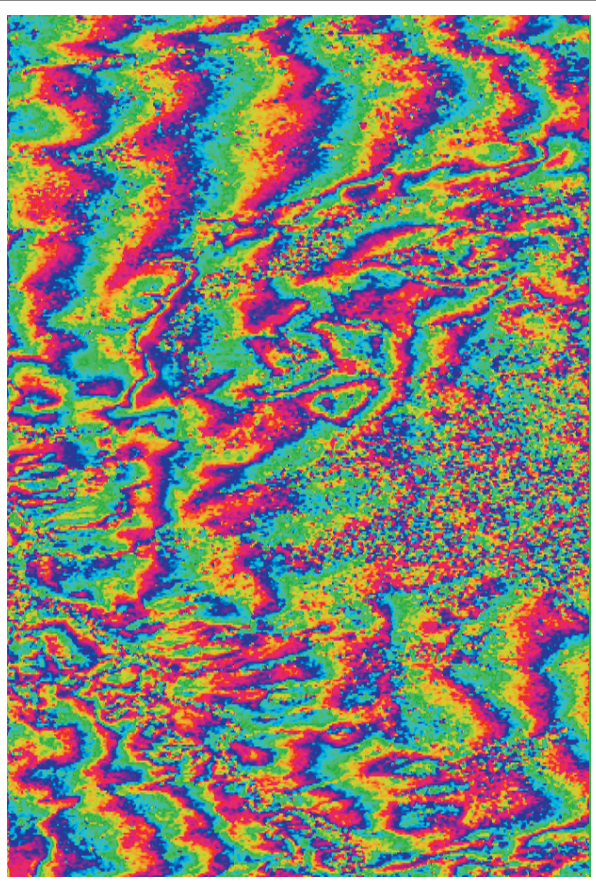

(b) Using DTED

Figure 7. Filtered residual RADARSAT-1 interferograms using various DEMs to flatten the phase. 


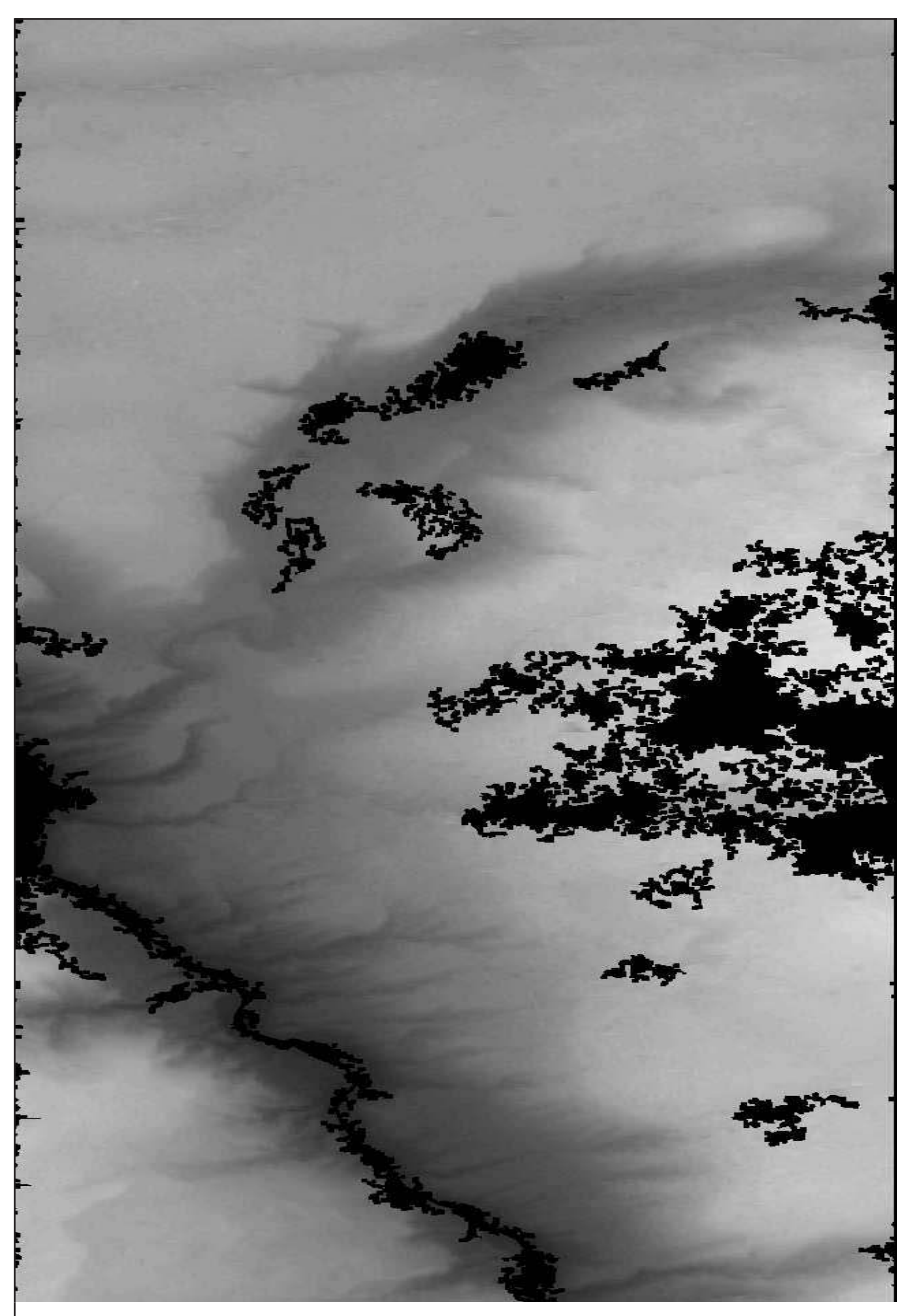

Figure 8. Updated TRIM DEM using RADARSAT-1 InSAR data.

\section{RADARSAT DEM refinement experiments}

The SAR data shown in Figure 2 were used to perform DEM refinement experiments. The DEMs of Figure 3 were used with the baseline estimated from the "interferogram conditioning" procedure to generate models of the interferogram phase. The modelled phase was subtracted from the measured interferogram phase of Figure $\mathbf{2 b}$, then filtered to get the residual interferograms shown in Figure 7. In effect, the information in the coarse DEM has been used to "flatten" the measured interferogram and make a first estimate of the baseline parameters. This flattening is much more complete than what is usually obtained with normal "flat-earth" flattening; for comparison, the phase after conventional flattening is shown in Figure $2 \mathbf{d}$.

The improved flattening makes the residual interferogram easier to filter and subsequently unwrap. Because the DEMflattened interferograms have a reduced bandwidth compared with the original interferogram, a greater degree of phase smoothing (bandpass filtering) can be applied to make phase unwrapping more reliable. However, the bandpass filtering may also eliminate some detail, so the amount of filtering must be selected to obtain the best tradeoff between suppressing interferogram noise and conserving DEM detail.

The residual phase of Figure 7 represents the radar information missing from the input DEMs. This phase is used to update the DEMs, and the results are shown in Figures 8-10. The black areas in the output DEMs correspond to areas of low coherence where valid height estimates could not be calculated from the InSAR data. These areas were masked to eliminate them from the DEM updating processing. A higher coherence dataset would have few or none of these unusable areas.

In addition to using the DEMs directly, modified versions of the DTED-1 and GTOPO30 DEMs were used as algorithm inputs, in which the TRIM data were used to remove biases and linear trends from these DEMs and to correct some obvious phase unwrapping errors in the GTOPO30 case. The resulting height errors are summarized in Table 4. The error statistics were calculated from the difference between the updated DEM and the TRIM input DEM, using all the valid pixels in the DEMs. The 90th percentile error was calculated from this error after removing the mean value.

Results with the TRIM data are shown in Figure 8. Some additional detail has been added to the TRIM DEM, but we have little basis upon which to assess the improvement, as the TRIM data are our only ground truth. The standard deviation of $12 \mathrm{~m}$ between the original and updated TRIM DEMs could represent errors in the original TRIM data, e.g., due to undersampling; however, it could also represent errors due to incorrect baseline estimates and interferogram noise, as the input TRIM data have a claimed error of $3 \mathrm{~m} \mathrm{rms}$. Assuming that the majority of the errors come from interferogram noise and baseline errors, the $12 \mathrm{~m}$ rms value serves as a lower limit of DEM improvement with the current radar dataset.

For the DTED-1 results in Figure 9, the majority of the data were unwrapped correctly and reasonable improvement of the initial input DEM was possible. We can see that extra detail has been added to the DTED-1 data, particularly in the region of the river canyons. The DEM standard deviation was lowered from $36 \mathrm{~m}$ (input DEM versus TRIM) to $19 \mathrm{~m}$ (output DEM versus TRIM). After removing the bias and trends in the input DEM, the standard deviation of the updated DTED-1 DEM was reduced further to $14 \mathrm{~m}$. In this case, the output product approaches the TRIM data quality (as it is close to the $12 \mathrm{~m}$ limit discussed in the preceding paragraph) and likely has an accuracy compatible with that of the DTED-2 standard.

The GTOPO30 dataset shown in Figure 10a does not perform as well in its unmodified form. There are a number of different phase groups that are not unwrapped contiguously. These errors are due to the coarse nature of the input DEM, which generates correlated errors biased low on the west side of the Fraser River. After removing the phase unwrapping errors and the bias and trend errors in the initial DEM, we get results reasonably close to those of the input TRIM DEM at $19 \mathrm{~m}$ standard deviation. 
The consistent limit of achieved accuracy (the values 19, 20, and $21 \mathrm{~m}$ in the last column of Table 4) suggests that the algorithm is extracting all the useful information in the InSAR dataset. It shows the algorithm is consistent and relatively insensitive to the quality of the DEM data as long as it has moderate offset and trend errors.

\section{Conclusions}

We have developed a new method of using existing DEM data to improve the calibration of satellite InSAR data and to make the processing more reliable. This allows us to use the InSAR data to add detail to the existing DEMs in a robust and

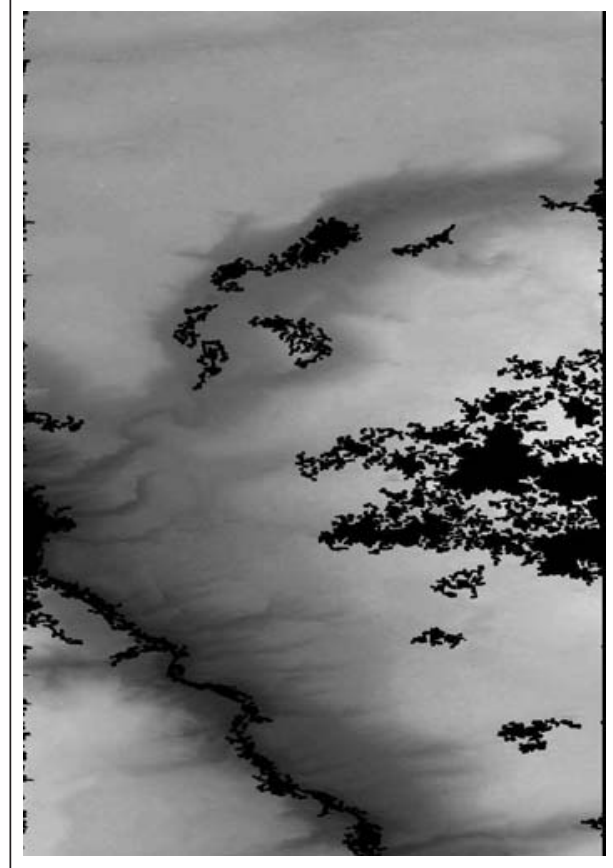

(a) Output DTED DEM

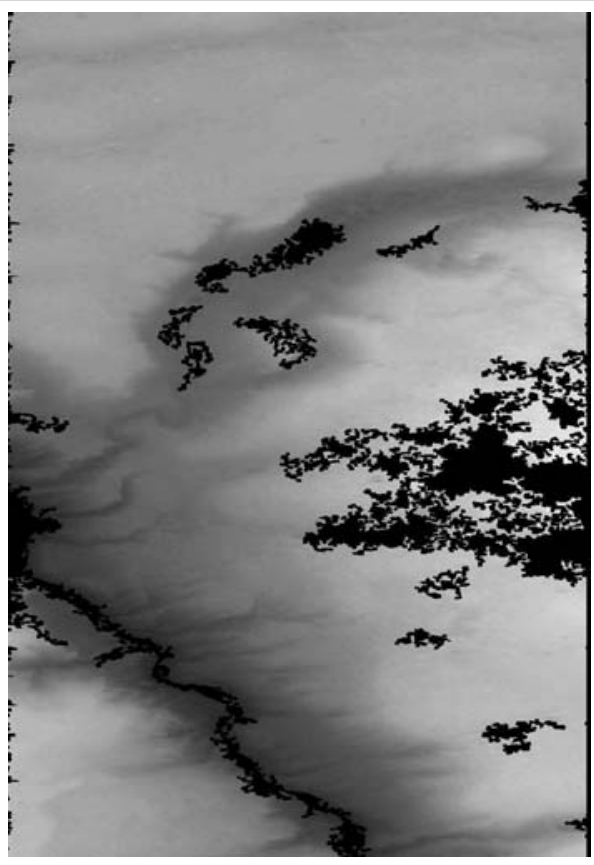

(b) Modified output DTED DEM

Figure 9. Updated DTED-1 DEM using RADARSAT-1 InSAR data.

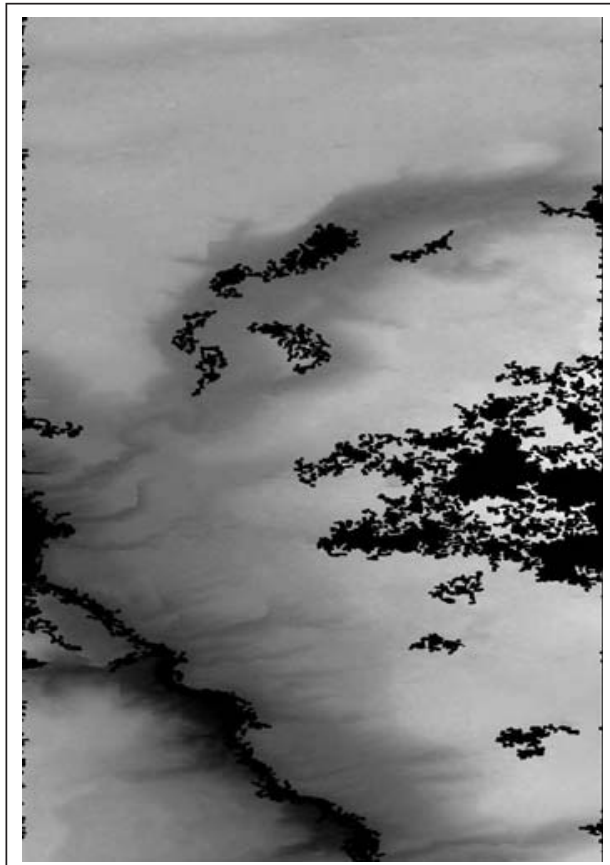

(a) Output GTOPO30 DEM

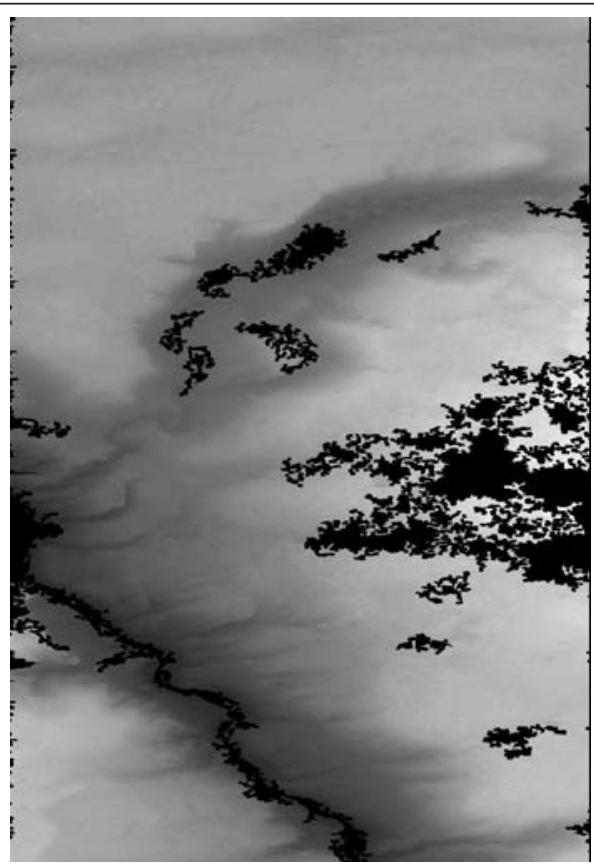

(b) Modified output GTOPO30 DEM

Figure 10. Updated GTOPO30 DEM using RADARSAT-1 InSAR data. 
Table 4. Statistics of the input and output (updated) DEMs in comparison with the reference TRIM DEM.

\begin{tabular}{|c|c|c|c|c|c|c|}
\hline \multirow[b]{2}{*}{ DEM } & \multicolumn{3}{|l|}{ Input (m) } & \multicolumn{3}{|c|}{ Output (m) } \\
\hline & $\mu$ & $\sigma$ & $\begin{array}{l}\text { 90th percentile } \\
\text { error }\end{array}$ & $\mu$ & $\sigma$ & $\begin{array}{l}\text { 90th percentile } \\
\text { error }\end{array}$ \\
\hline TRIM & Ground truth & Ground truth & Ground truth & -0.4 & 12 & 19 \\
\hline DTED-1 & -16.0 & 36 & 61 & -17.0 & 19 & 37 \\
\hline Modified DTED-1 & -0.1 & 35 & 57 & -0.6 & 14 & 20 \\
\hline GTOPO30 & 7.8 & 98 & 144 & 6.8 & 64 & 112 \\
\hline Modified GTOPO30 & 0.1 & 91 & 134 & -0.8 & 19 & 21 \\
\hline
\end{tabular}

largely automatic manner, as long as atmospheric phase errors are not too large.

The DEM refinement algorithm was applied to RADARSAT1 InSAR data using DEMs of varying quality. These included good quality (TRIM), moderate quality (DTED-1), and poor quality (GTOPO30) DEMs. For all input DEMs, the interferogram conditioning procedure was able to provide a residual interferogram with substantially less phase variability than the originally measured interferogram. This facilitated phase unwrapping through filtering and subsampling of the residual interferogram.

The DEM updating procedure was successfully applied to the various DEMs, although manual intervention was needed for the very coarse GTOPO30 data. Using the RADARSAT-1 data, we were able to substantially improve the quality of the input DTED-1 (roughly halving the input standard deviation) in both the modified and unmodified DEM cases. For the GTOPO30 dataset, there were phase unwrapping discontinuities between different unwrapped phase groups, which caused a recognizable failure in the algorithm's operations. After the phase unwrapping errors had been corrected and the input DEM compensated for bias and trend errors, the results were on the order of a factor of four better than in the input DEM. The sensitivity of the DEM refinement algorithm to input DEM bias and trend errors was demonstrated, as the changes in output mirrored the changes in the trends and biases in the input DEM.

Our test case was very challenging because of the steep topography and low coherence and because of recognizable errors in the input DEMs. We expect that better results could be achieved with better quality InSAR and DEM data. In particular, the public domain DEMs available from the successful Shuttle Radar Topography Mission (SRTM; Hilland et al., 1998) could be improved by taking advantage of the large amount of archived two-pass satellite SAR data, which have a finer grid spacing than the SRTM DEM data.

\section{Acknowledgments}

The authors wish to thank Roger Balser of the British Columbia Mapping Branch for providing the TRIM data and the Application Development and Research Opportunity (ADRO) program for the RADARSAT data. Financial support from the Natural Sciences and Engineering Research Council of Canada (NSERC), MacDonald Dettwiler, and the BC Advanced Systems Institute is gratefully acknowledged.

\section{References}

Davidson, G.W., and Bamler, R. 1999. Multiresolution phase unwrapping for SAR interferometry. IEEE Transactions on Geoscience and Remote Sensing, Vol. 37, pp. 163-174.

Dupont, S. 1997. Génération de modèle numérique de terrain par interférométrie ROS (SAR). Ph.D. thesis, Université de Nice Sophia Antipolis, Nice, France.

Eineder, M. 2003. Efficient simulation of SAR interferograms of large areas and of rugged terrain. IEEE Transactions on Geoscience and Remote Sensing, Vol. 41, No. 6, pp. 1415-1427.

Ferretti, A., Prati, C., and Rocca, F. 1999. Multibaseline InSAR DEM reconstruction: the wavelet approach. IEEE Transactions on Geoscience and Remote Sensing, Vol. 37, pp. 705-715.

Flynn, T.J. 1997. Two-dimensional phase unwrapping with minimum weighted discontinuity. Journal of the Optical Society of America A, Vol. 14, pp. 2692-2701.

Gesch, D.B. 1998. Accuracy assessment of a global elevation model using shuttle laser altimetry data. In IGARSS'98, Proceedings of the IEEE International Geoscience and Remote Sensing Symposium, 6-10 July 1998, Seattle, Wash. IEEE, New York. Vol. 2, pp. 840-842.

Geudtner, D., and Schwabisch, M. 1996. An algorithm for precise reconstruction of InSAR imaging geometry: application to "flat earth" phase removal, phase-to-height conversion, and geocoding of InSARderived DEMs. In EUSAR'96, Proceedings of the European Conference on Synthetic Aperture Radar, 26-28 March 1996, Konigswinter, Germany. pp. 249-252.

Ghiglia, D.C., and Pritt, M.D. 1998. Two-dimensional phase unwrapping: theory, algorithms, and software. John Wiley and Sons, New York.

Ghiglia, D., and Romero, L. 1994. Robust two-dimensional weighted and unweighted phase wrapping that uses fast transforms and iterative methods. Journal of the Optical Society of America A, Vol. 11, pp. 107-117.

Goldstein, R. 1995. Atmospheric limitations to repeat pass interferometry. Geophysical Research Letters, Vol. 22, pp. 2517-2520.

Graham, L.C. 1974. Satellite interferometer radar for topographic mapping. Proceedings of the IEEE, Vol. 62, No. 6. pp. 763-768.

Gray, A., Mattar, K., and Sofko, G. 2000. Influence of ionospheric electron density fluctuations on satellite radar interferometry. Geophysical Research Letters, Vol. 27, pp. 1451-1454. 
Hanssen, R. 1997. Atmospheric heterogeneities in ERS tandem SAR interferometry. Ph.D. thesis, Delft University of Technology, Delft, The Netherlands.

Hanssen, R., Weinreich, I., Lehner, S., and Stoffelen, A. 2000. Tropospheric wind and humidity derived from spaceborne radar intensity and phase observations. Geophysical Research Letters, Vol. 27, pp. 1699-1702.

Hilland, J., Stuhr, F., Freeman, A., Imel, D., Shen, Y., Jordan, R., and Caro, E. 1998. Future NASA spaceborne SAR missions. IEEE Aerospace and Electronics Systems Magazine, Vol. 13, pp. 9-16.

Massonnet, D. 1997. Producing ground deformation maps automatically: the DIAPASON concept. In IGARSS'97, Proceedings of the IEEE International Geoscience and Remote Sensing Symposium, 3-8 August 1997, Singapore. IEEE, New York. Vol. 3, pp. 1338-1340.

Massonnet, D., and Vadon, H. 1993. Precision positioning of interferometric track. In Proceedings of the CEOS SAR Calibration Workshop, 20-24 September 1993, Noordwijk, The Netherlands. European Space Agency (ESA) Publications Division, Noordwijk, The Netherlands. ESA Publication WPP-048.

Massonnet, D., Rossl, M., Carmona, C., Adragna, F., Peltzer, G., Feigl, K., and Rabaute, T. 1993. The displacement field of the Landers earthquake mapped by radar interferometry. Nature (London), Vol. 364, pp. 138-142.

Mattar, K.E., and Gray, A.L. 2002. Reducing ionospheric electron density errors in satellite radar interferometry applications. Canadian Journal of Remote Sensing, Vol. 28, No. 4, pp. 593-600.

Mattar, K.E., Gray, A.L., Geudtner, D., and Vachon, P.W. 1999. Interferometry for DEM and terrain displacement: effects of inhomogenous propagation. Canadian Journal of Remote Sensing, Vol. 25, No. 1, pp. 60-69.

Prati, C., and Rocca, F. 1992. Range resolution enhancement with multiple SAR surveys used in combination. In IGARSS'92, Proceedings of the IEEE International Geoscience and Remote Sensing Symposium, 26-29 May 1992, Clear Lake, Tex. IEEE, New York. pp. 1576-1578.

Schwabisch, M. 1998. Large scale interferometric DEM generation using ERS tandem data: example of the Czech Republic. In IGARSS'98, Proceedings of the IEEE International Geoscience and Remote Sensing Symposium, 610 July 1998, Seattle, Wash. IEEE, New York.

Seymour, M. 1999. Refining low-quality digital elevation models using synthetic aperture radar interferometry. Ph.D. thesis, University of British Columbia, Vancouver, B.C.

Seymour, M., and Cumming, I. 1997. InSAR terrain height estimation using low-quality sparse DEMs. In Proceedings of the 3rd ERS Scientific Symposium, 17-21 March 1997, Florence, Italy. Edited by G. Kohlhammer, T.D. Guyenne, and D. Danesy. European Space Agency (ESA) Publications Division, Noordwijk, The Netherlands. ESA Publication SP-414. Vol. 1, pp. $421-426$.

Surveys and Resources Mapping Branch. 1992. British Columbia specifications and guidelines for geomatics. Release 2.0 edition. Surveys and Resources Mapping Branch, Ministry of Environment, Lands and Parks, Province of British Columbia, Victoria, B.C.

Tarayre, H., and Massonnet, D. 1994. Effects of a refractive atmosphere on interferometric processing. In IGARSS'94, Proceedings of the IEEE International Geoscience and Remote Sensing Symposium, 8-12 August 1994, Pasadena, Calif. IEEE, New York. pp. 717-719.

Tarayre, H., and Massonnet, D. 1996. Atmospheric propagation heterogeneities revealed by ERS-1 interferometry. Geophysical Research Letters, Vol. 23, pp. 989-992.
Vachon, P.W., Geudtner, D., Gray, A., and Touzi, R. 1995. ERS-1 synthetic aperture repeat-pass interferometry: implications for RADARSAT. Canadian Journal of Remote Sensing, Vol. 21, No. 4, pp. 441-454.

van der Kooij, M.W.A., Armour, B., Ehrismann, J., Schwichow, H., and Sato, S. 1996. A workstation for spaceborne interferometric SAR data. In IGARSS'96, Proceedings of the IEEE International Geoscience and Remote Sensing Symposium, 27-31 May 1996, Lincoln, Nebr. IEEE, New York. Vol. 1, pp. 339-341.

Wegmüller, U., and Werner, C. 1997. Gamma SAR processor and interferometry software. In Proceedings of the 3rd ERS Scientific Symposium, 17-21 March 1997, Florence, Italy. Edited by G. Kohlhammer, T.D. Guyenne, and D. Danesy. European Space Agency (ESA) Publications Division, Noordwijk, The Netherlands. ESA Publication SP-414. Vol. 3, pp. 1687-1692.

Zebker, H.A., and Goldstein, R.M. 1986. Topographic mapping from interferometric synthetic aperture radar observations. Journal of Geophysical Research, Vol. 91, No. B5, pp. 4993-4999.

Zebker, H., and Villasenor, J. 1992. Decorrelation in interferometric radar echoes. IEEE Transactions on Geoscience and Remote Sensing, Vol. 30, pp. 950-959.

Zebker, H.A., Werner, C.L., Rosen, P.A., and Hensley, S. 1994. Accuracy of topographic maps derived from ERS-1 interferometric radar. IEEE Transactions on Geoscience and Remote Sensing, Vol. 32, pp. 823-836.

\section{Appendix A. Plane fits to random noise}

One way to derive the best possible topographic accuracy achievable for an input DEM is to analyze the experiment of fitting a linear model of the DEM errors due to baseline parameter errors to a set of random, zero-mean, independent, and identically distributed random variables with variance $\sigma^{2}$. This models the ideal case of DEM fitting.

The problem is to minimize:

$\sum(a+b y+c x+d x y-\Delta h)^{2}=\left\|\mathbf{A}\left[\begin{array}{l}a \\ b \\ c \\ d\end{array}\right]-\boldsymbol{h}\right\|^{2}$

where $a$ models the constant error of the output DEM; $b$ models the range slope error of the output DEM; $c$ models the azimuth slope error of the output DEM; $d$ models the bilinear trend error of the output DEM; $\Delta h$ models the errors of the input coarse DEM; $\mathbf{A}$ is the matrix of fitting coefficients; $\boldsymbol{h}$ is the DEM errors in a vector; and

$$
\begin{array}{ll}
y=k-\frac{\left(N_{y}-1\right)}{2}, & k=0 \ldots N_{y}-1, \\
x=l-\frac{\left(N_{x}-1\right)}{2}, & l=0 \ldots N_{x}-1
\end{array}
$$

The solution to the minimization problem is the usual least squares solution: 
$\left[\begin{array}{l}a \\ b \\ c \\ d\end{array}\right]=\left(\mathbf{A}^{\mathrm{T}} \mathbf{A}\right)^{-1} \mathbf{A}^{\mathrm{T}} \boldsymbol{h}$.

Since we have taken care to choose our fitting coefficients to be orthogonal, $\left(\mathbf{A}^{\mathrm{T}} \mathbf{A}\right)^{-1}$ takes a simple diagonal form:

$$
\left(\mathbf{A}^{\mathrm{T}} \mathbf{A}\right)^{-1}=\left[\begin{array}{ccc}
\frac{1}{N_{x} N_{y}} & & \\
& \frac{12}{N_{x} N_{y}\left(N_{y}^{2}-1\right)} & \\
& \frac{12}{N_{y} N_{x}\left(N_{x}^{2}-1\right)} & 144 \\
& & \frac{1}{N_{x}^{3} N_{y}^{3}-N_{x}^{3} N_{y}-N_{y}^{3} N_{x}+N_{x} N_{y}}
\end{array}\right] .
$$

The values of the coefficients are therefore

$$
a=\frac{1}{N_{x} N_{y}} \sum_{k, l} \Delta h_{k, l}
$$$$
b=\frac{12}{N_{x} N_{y}\left(N_{y}^{2}-1\right)} \sum_{k, l} \Delta h_{k, l}\left(k-\frac{N_{y}-1}{2}\right) \text {, }
$$$$
c=\frac{12}{N_{y} N_{x}\left(N_{x}^{2}-1\right)} \sum_{k, l} \Delta h_{k, l}\left(l-\frac{N_{l}-1}{2}\right),
$$

$$
\begin{aligned}
d= & \frac{144}{N_{x}^{3} N_{y}^{3}-N_{x}^{3} N_{y}-N_{y}^{3} N_{x}+N_{x} N_{y}} \\
& \times \sum_{k, l} \Delta h_{k, l}\left(k-\frac{N_{y}-1}{2}\right)\left(l-\frac{N_{l}-1}{2}\right) .
\end{aligned}
$$

If the noise variables are independent and zero mean, then it is clear that the expected value of all the fitted variables is zero, i.e.,

$\mathrm{E}[a]=0, \mathrm{E}[b]=0, \mathrm{E}[c]=0, \mathrm{E}[d]=0$.

A corollary of this result is that the output DEM will not (in the mean sense) have any trends. In addition, because the basis functions that are being fitted are orthogonal, there is no correlation between the parameters, i.e., $\mathrm{E}[a b]=0$.

The variance of the parameters may be calculated by taking the expectation of the previous expressions. After some algebra, it can be shown that

$\mathrm{E}\left[a^{2}\right]=\frac{\sigma^{2}}{N_{x} N_{y}}$

$\mathrm{E}\left[b^{2}\right]=\frac{12 \sigma^{2}}{N_{x} N_{y}\left(N_{y}^{2}-1\right)}$,

$$
\mathrm{E}\left[c^{2}\right]=\frac{12 \sigma^{2}}{N_{x} N_{y}\left(N_{x}^{2}-1\right)},
$$

$$
\mathrm{E}\left[d^{2}\right]=\frac{144 \sigma^{2}}{N_{x}^{2} N_{y}^{3}-N_{y}^{3} N_{x}-N_{x}^{3} N_{y}+N_{x} N_{y}} .
$$

The variance of the output plane also establishes bounds on what the best-fitting interferometric DEM could achieve. Because the fitted plane has zero mean, one must evaluate

$\frac{1}{N_{x} N_{y}} \mathrm{E}\left[\sum \sum(b y+c x+d x y)^{2}\right]$.

Again, after some algebra, one can show that

$$
\frac{1}{N_{x} N_{y}} \mathrm{E}\left[\sum \sum(b y+c x+d x y)^{2}\right]=\frac{3 \sigma^{2}}{N_{x} N_{y}} \text {. }
$$

The variance of the height difference across the dataset is of interest because it gives an idea of the possible output trends in the InSAR DEM. One can calculate this as

$$
\begin{aligned}
\mathrm{E}\left\{\left[h\left(x_{2}, y_{2} \mid a, b, c, d\right)-h\left(x_{1}, y_{1} \mid a, b, c, d\right)\right]^{2}\right\} \\
\quad=\mathrm{E}\left\{\left[a+b y_{2}+c x_{2}+d x_{2} y_{2}\right.\right. \\
\left.\left.\quad-\left(a+b y_{2}+c x_{2}+d x_{2} y_{2}\right)\right]^{2}\right\} .
\end{aligned}
$$

Simplifying this expression by substituting for the differences yields

$$
\begin{gathered}
\mathrm{E}\left\{\left[b\left(y_{2}-y_{1}\right)+c\left(x_{2}-x_{1}\right)+d\left(x_{2} y_{2}-x_{1} y_{1}\right)\right]^{2}\right\} \\
=\mathrm{E}\left\{[b \Delta y+c \Delta x+d \Delta x y]^{2}\right\}
\end{gathered}
$$


where $\Delta y=y_{2}-y_{1}, \Delta x=x_{2}-x_{1}$, and $\Delta x y=x_{2} y_{2}-x_{1} y_{1}$. Expanding Equation (A10) and using the orthogonality of the basis functions yields

$$
\begin{aligned}
& \mathrm{E}\left\{[b \Delta y+c \Delta x+d \Delta x y]^{2}\right\}=\mathrm{E}\left[b^{2}\right] \Delta y^{2}+\mathrm{E}\left[c^{2}\right] \Delta x^{2} \\
& +\mathrm{E}\left[d^{2}\right] \Delta x y^{2} .
\end{aligned}
$$

Substituting for the expectation operators yields

$$
\mathrm{E}\left\{\left[h\left(x_{2}, y_{2} \mid a, b, c, d\right)-h\left(x_{1}, y_{1} \mid a, b, c, d\right)\right]^{2}\right\}
$$

$$
\begin{aligned}
& =\frac{12 \sigma^{2}}{N_{x} N_{y}\left(N_{y}^{2}-1\right)} \Delta y^{2}+\frac{12 \sigma^{2}}{N_{y} N_{x}\left(N_{x}^{2}-1\right)} \Delta x^{2} \\
& +\frac{144 \sigma^{2}}{N_{x}^{3} N_{y}^{3}-N_{x}^{3} N_{y}-N_{y}^{3} N_{x}+N_{x} N_{y}} \Delta x y^{2} .
\end{aligned}
$$

The maximum error possible occurs as a function of the relative position of the two points examined. There are six possible directions that could have the maximum values (four directions from one corner to the next along an edge, and two differences diagonally). However, one need only check diagonal direction and one edge to edge direction to get the form of the error.

For the difference across the fitted DEM diagonally we have

$\Delta x=N_{x}-1$

$\Delta y=N_{y}-1$,

$\Delta x y=0$,

and

$\mathrm{E}\left\{\left[h\left(x_{2}, y_{2} \mid a, b, c, d\right)-h\left(x_{1}, y_{1} \mid a, b, c, d\right)\right]^{2}\right\}_{\text {diag }}$

$$
=\frac{12 \sigma^{2}\left(N_{y}-1\right)}{N_{x} N_{y}\left(N_{y}+1\right)}+\frac{12 \sigma^{2}\left(N_{x}-1\right)}{N_{y} N_{x}\left(N_{x}+1\right)} .
$$

For the DEM difference across an edge, we evaluate the error in the difference between the two heights at the corners along the right edge of the DEM, where we have

$$
\begin{aligned}
\Delta x= & 0, \\
\Delta y= & N_{y}-1, \\
\Delta x y= & \frac{\left(N_{x}-1\right)}{2} \frac{\left(N_{y}-1\right)}{2} \\
& -\frac{\left(N_{x}-1\right)}{2}\left[-\frac{\left(N_{y}-1\right)}{2}\right]=\frac{\left(N_{x}-1\right)\left(N_{y}-1\right)}{2} .
\end{aligned}
$$

$\mathrm{E}\left\{\left[h\left(x_{2}, y_{2} \mid a, b, c, d\right)-h\left(x_{1}, y_{1} \mid a, b, c, d\right)\right]^{2}\right\}_{\text {right }}$

$$
\begin{aligned}
& =\frac{12 \sigma^{2}\left(N_{y}-1\right)}{N_{x} N_{y}\left(N_{y}+1\right)} \\
& +\frac{144 \sigma^{2}}{N_{x}^{3} N_{y}^{3}-N_{x}^{3} N_{y}-N_{y}^{3} N_{x}+N_{x} N_{y}}
\end{aligned}
$$

$$
\times \frac{\left(N_{x}-1\right)^{2}\left(N_{y}-1\right)^{2}}{4}
$$

This simplifies to

$\mathrm{E}\left\{\left[h\left(x_{2}, y_{2} \mid a, b, c, d\right)-h\left(x_{1}, y_{1} \mid a, b, c, d\right)\right]^{2}\right\}_{\text {right }}$

$$
=\frac{12 \sigma^{2}\left(N_{y}-1\right)}{N_{x} N_{y}\left(N_{y}+1\right)}+\frac{36 \sigma^{2}\left(N_{y}-1\right)\left(N_{x}+1\right)}{N_{x} N_{y}\left(N_{x} N_{y}+N_{y}+N_{x}+1\right)} .
$$

For $N_{y}=N_{x}=N$, the edge difference will be more than the difference diagonally, which suggests that the bounds for error performance of the estimate are really set along the edge of the DEM as

$\mathrm{E}\left\{\left[h\left(x_{2}, y_{2} \mid a, b, c, d\right)-h\left(x_{1}, y_{1} \mid a, b, c, d\right)\right]^{2}\right\}_{\text {right }}$

$$
=\frac{24 \sigma^{2}(2 N-1)(N-1)}{(N+1)^{2} N^{2}} .
$$

and 